Age $<20$ years was associated with NMDA-R-IgG and MOG-IgG1 $(\mathrm{OR}=8.11$ and 7.73 respectively, $\mathrm{p}<0.001)$. Age $>65$ years was associated with GABAB-R-IgG, LGI1-IgG, CASPR2-IgG and ANNA1-IgG (OR=7.33, 14.98, 3.67, 14.53, $\mathrm{p}<0.001)$. Women accounted for $60 \%$ of NMDA-R-IgG (CSF) and $78 \%$ of GAD65-IgG (CSF/serum) cohorts $(\mathrm{OR}=1.32$, $\mathrm{p}=0.002, \mathrm{OR}=2.78, \mathrm{p}<0.001$, respectively). Men accounted for $62 \%$ of the LGI1-IgG cohort $(\mathrm{OR}=1.87, \mathrm{p}<0.001)$. Age and sex interacted for NMDA-R-IgG, particularly in females $<20$ years $(\mathrm{OR}=7.72, \mathrm{p}<0.001)$.

Conclusion The most frequently detected were NMDA-R-IgG, LGI1-IgG, GAD65-IgG and MOG-IgG1. Age and sex associations may suggest paraneoplastic, endocrinological or aging influences on neurological autoimmunity.

\section{PREGNANCY-RELATED RELAPSE IN NATALIZUMAB, FINGOLIMOD AND DIMETHYL FUMARATE-TREATED WOMEN WITH MULTIPLE SCLEROSIS}

1,2Wei Z Yeh, ${ }^{1}$ Putu A Widyastuti, ${ }^{1,2}$ Anneke Van der Walt, ${ }^{1}$ Jim Stankovich, ${ }^{3}$ Eva K Havrdova, ${ }^{3}$ Dana Horakova, ${ }^{3}$ Karolina Vodehnalova, ${ }^{4}$ Serkan Ozakbas, ${ }^{5}$ Sara Eichau, ${ }^{6}$ Pierre Duquette, ${ }^{8,7}$ Tomas Kalincik, ${ }^{9}$ Francesco Patti, ${ }^{10}$ Cavit Boz, ${ }^{11}$ Murat Terzi, ${ }^{12}$ Bassem Yamout, ${ }^{13}$ Jeannette Lechner-Scott, ${ }^{14}$ Patrizia Sola, ${ }^{2}$ Olga Skibina, ${ }^{15}$ Michael Barnett, ${ }^{16}$ Marco Onofrj, ${ }^{17}$ Maria J Sá, ${ }^{18,19}$ Pamela McCombe, ${ }^{20}$ Pierre Grammond, ${ }^{21}$ Radek Ampapa, ${ }^{22}$ Francois Grand'Maison, ${ }^{23}$ Roberto Bergamaschi, ${ }^{24}$ Daniele LA Spitaleri, ${ }^{25}$ Vincent Van Pesch, ${ }^{26}$ Elisabetta Cartechini, ${ }^{27}$ Suzanne Hodgkinson, ${ }^{28}$ Aysun Soysal, ${ }^{29}$ Albert Saiz, ${ }^{1,2}$ Melissa Gresle, ${ }^{3}$ Tomas Uher, ${ }^{30}$ Davide Maimone, ${ }^{31}$ Recai Turkoglu, ${ }^{32}$ Raymond MM Hupperts, ${ }^{33,34}$ Maria Pia Amato, ${ }^{35}$ Franco Granella, ${ }^{36}$ Celia Oreja-Guevara, ${ }^{37}$ Ayse Altintas, ${ }^{38}$ Richard Macdonell, ${ }^{39}$ Tamara Castillo-Trivino, ${ }^{1,2}$ Helmut Butzkueven, ${ }^{40}$ Raed Alroughani, ${ }^{1,2}$ Vilija G Jokubaitis, ${ }^{41}$ MSBase Registry. ${ }^{1}$ Department of Neuroscience, Central Clinical School, Monash University, Melbourne, VIC, Australia; ${ }^{2}$ Multiple Sclerosis and Neuroimmunology Unit, Department of Neurology, Alfred Health, Melbourne, VIC, Australia; ${ }^{3}$ Department of Neurology and Center of Clinical Neuroscience, First Faculty of Medicine, Charles University and General University Hospital, Prague, Czech Republic; ${ }^{4}$ Dokuz Eylul University, Turkey; ${ }^{5}$ Hospital Universitario Virgen Macarena, Spain; ${ }^{6}$ CHUM - Hopital Notre Dame, Canada; ${ }^{7}$ Melbourne MS Centre, Royal Melbourne Hospital, Melbourne, VIC, Australia; ${ }^{8} \mathrm{CORe}$, Department of Medicine, University of Melbourne, Melbourne, VIC, Australia; ${ }^{9}$ Department of Medical and Surgical Sciences and Advanced Technologies; GF Ingrassia, University of Catania - AOU Policlinico-San Marco, University of Catania, Italy; ${ }^{10}$ KTU Medical Faculty Farabi Hospital, Turkey; ${ }^{11}$ Mayis University, Medical Faculty, Turkey; ${ }^{12}$ American University of Beirut, Faculty of Medicine, Nehme and Therese Multiple Sclerosis Center, Beirut, Lebanon; ${ }^{13}$ John Hunter Hospital, New Lambton Heights, NSW, Australia; ${ }^{14}$ Neurology Unit, Azienda Ospedaliero-Universitaria of Modena, Modena, Italy; ${ }^{15}$ Brain and Mind Centre, University of Sydney, Sydney, NSW, Australia; ${ }^{16}$ Univ G.d'Annunzio Chieti-Pescara, Italy; ${ }^{17}$ Department of Neurology, São João Universitary Hospital Center, Porto, Portugal; ${ }^{18}$ Royal Brisbane and Women's Hospital, Herston, QLD, Australia; ${ }^{19}$ St Andrews Place, Spring Hill, QLD, Australia; ${ }^{20}$ Centre de réadaptation déficience physique Chaudière-Appalache, Canada; ${ }^{21}$ Nemocnice Jihlava, Czech Republic; ${ }^{22}$ Neuro Rive-Sud, Canada; ${ }^{23}$ IRCCS Mondino Foundation, Pavia, Italy; ${ }^{24}$ AORN San Giuseppe Moscati Avellino, Italy; ${ }^{25}$ Cliniques Universitaires Saint-Luc, Université Catholique de Louvain, Belgium; ${ }^{26}$ Ospedale Generale Provinciale Macerata, Italy; ${ }^{27}$ Liverpool Hospital, Liverpool, NSW, Australia; ${ }^{28}$ Bakirkoy Education and Research Hospital for Psychiatric and Neurological Diseases, Turkey; ${ }^{29}$ Service of Neurology, Hospital Clinic, Institut d'Investigacions Biomediques August Pi i Sunyer (IDIBAPS), and Institut de Neurociències, Universitat de Barcelona, Barcelona, Spain; ${ }^{30}$ Centro Sclerosi Multipla, UOC Neurologia, ARNAS Garibaldi, Catania, Italy; ${ }^{31}$ Haydarpasa Numune Training and Research Hospital, Turkey; ${ }^{32}$ Maaslandziekenhuis, Netherlands; ${ }^{33}$ Department NEUROFARBA, University of Florence, Italy; ${ }^{34}$ IRCCS Fondazione Don Carlo Gnocchi, Florence, Italy; ${ }^{35}$ University of Parma, Italy; ${ }^{36}$ Department of Neurology, Hospital Clínico San Carlos, Departamento de Medicina, Facultad de Medicina, Universidad Complutense de Madrid (UCM) and IdISSC, Madrid, Spain; ${ }^{37}$ Department of Neurology, Koc University School of Medicine, Turkey; ${ }^{38}$ Department of Neurology, Austin Health, Heidelberg, VIC, Australia; ${ }^{39}$ Department of Neurology, Hospital Universitario Donostia, San Sebastian, Spain; ${ }^{40}$ Amiri Hospital, Kuwait; ${ }^{41}$ MSBase Neuro-immunology Registry, Melbourne, VIC, Australia

10.1136/bmjno-2021-ANZAN.4
Objective To investigate pregnancy-related disease activity in a contemporary multiple sclerosis (MS) cohort.

Methods Data were obtained from the MSBase Registry. Term/ preterm pregnancies conceived from 2011-2019 were included (modern cohort). Annualised relapse rates (ARR) were calculated before, during and after pregnancy. Predictors of intrapartum and early postpartum ( $1^{\text {st }} 3$ months) relapse were determined by clustered logistic and Cox regression analyses, respectively.

Results We included 1640 pregnancies from 1452 women. Disease-modifying therapy (DMT) used in the one-year preconception included natalizumab $(n=219)$, fingolimod $(n=147)$, dimethyl fumarate (DMF; $n=57)$ and low-efficacy therapies $(n=845)$. Preconception ARR by DMT class used before conception were: natalizumab, 0.29 (95\% CI 0.22 0.37); fingolimod, 0.37 (0.28-0.49); DMF, 0.24 (0.13-0.41); low-efficacy, 0.29 (0.25-0.33); and none, 0.24 (0.19-0.31). Among women who used fingolimod or natalizumab, ARR increased during pregnancy. Intrapartum ARR decreased in preconception DMF, low-efficacy or no DMT groups. ARR spiked after delivery across all DMT groups. Natalizumab continuation into pregnancy reduced the odds of relapse during pregnancy (OR 0.76 per month [0.60-0.95], $\mathrm{p}=0.017$ ). DMT re-initiation with natalizumab protected against postpartum relapse (HR 0.11 [0.04-0.32], $\quad \mathrm{p}<0.0001)$. Breastfeeding women were less likely to relapse (HR 0.61 [0.41-0.91], $\mathrm{p}=0.016)$.

Conclusion Women with MS prescribed natalizumab or fingolimod preconception had higher rates of intrapartum and postpartum relapse. In women considered to be at high relapse risk, use of natalizumab before pregnancy and continued up to 32-34 weeks gestation, with early re-initiation after delivery is an effective option to minimise relapse risks. Strategies of DMT use have to be balanced against potential foetal/neonatal complications.

\section{PSORIASIS IN MULTIPLE SCLEROSIS: AN AUSTRALIAN PREVALENCE STUDY}

${ }^{1}$ Varitsara Mangkorntongsakul, ${ }^{2}$ Olivia A Charlton, ${ }^{3}$ Kevin Phan, ${ }^{4}$ Ariadna Fontes, ${ }^{1,4}$ John Parratt, ${ }^{1,4}$ Geoff Herkes, ${ }^{5,6,7}$ Saxon D Smith. ${ }^{1}$ The University of Sydney, Sydney, NSW, Australia; ${ }^{2}$ Department of Dermatology, Royal North Shore Hospital, St Leonards, NSW, Australia; ${ }^{3}$ Department of Dermatology, Liverpool Hospital, Liverpool, NSW, Australia; ${ }^{4}$ Department of Neurology, Royal North Shore Hospital, St Leonards, NSW, Australia; ${ }^{5}$ The Dermatology and Skin Cancer Centre, Gosford, NSW, Australia; ${ }^{6}$ Sydney Adventist Hospital Clinical School, Sydney Medical School, The University of Sydney, Sydney, NSW, Australia; 'Department of Dermatology, Sydney Adventist Hospital, Wahroonga, NSW, Australia

\subsection{6/bmjno-2021-ANZAN.5}

Background Multiple Sclerosis (MS) is an immune-mediated, demyelinating disease of the central nervous system. ${ }^{1}$ Although severe psoriasis and psoriasiform dermatitis have been noted in MS patients, the prevalence of psoriasis in these populations is uncertain and has not been explored in the Australian population.

Objectives A pilot study to estimate the prevalence of psoriasis in MS cohorts in the Australian population.

Methods A survey was conducted on 82 MS patients aged 18 and above who attended MS clinics in 2018.

Results Data was recorded for 82 patients. The mean age was 48 years for the entire cohort and 48.0 years (SD \pm 11.30 ) 